\title{
NEW APPROACHES ON DIMENSIONALITY REDUCTION IN HYPERSPECTRAL IMAGES FOR CLASSIFICATION PURPOSES
}

\author{
Daniele Cerra, Jakub Bieniarz, Rupert Mueller, and Peter Reinartz \\ Remote Sensing Technology Institute, \\ German Aerospace Center (DLR)
}

\begin{abstract}
This paper presents a quasi-unsupervised methodology to detect endmembers within an hyperspectral scene and to derive a pixel-wise classification on its basis. The endmember detection step takes as input an overcomplete spectral library, and detects the materials within a scene by analyzing derivative features under the sparsity assumption. The purest pixels for each detected material are then fed to a classifier based on synergetics theory, which is able to produce accurate classification maps on the basis of a restricted training dataset. As the classifier projects the image onto a subspace composed by the classes of interest found in the first step, a focused dimensionality reduction is performed in which every dimension is semantically meaningful.
\end{abstract}

Index Terms - Hyperspectral image classification, sparsity, spectral unmixing, synergetics, endmember detection.

\section{INTRODUCTION}

Hyperspectral data are characterized by very rich spectral information, which makes them apt to detecting targets of interest, but are affected by several specific drawbacks, caused by their high dimensionality and moderate spatial resolution. Firstly, the very high dimensionality of these data introduces a series of problems, summarized by the principle known as curse of dimensionality. As in most cases not all the bands are useful for a given application, a preliminary step of band selection can be performed. Alternatively (or in addition), the data are often projected on a lowerdimensionality space to aid data exploration and improve computation performances. This is usually a step preceding other operations such as classification and target detection [1]. Some of the most widely used dimension reduction techniques in remote sensing are based on principal component analysis (PCA) [2]. If the user desires to differentiate different classes of interest, however, such approaches may not be optimal, as in general the dimensions in the subspaces do not convey any semantics, and therefore may not match the user's needs. Another main drawback of hyperspectral sensors is their limited spatial resolution with respect to panchromatic or multispectral sensors, which causes the spectra associated with the image elements to be usually composed by a mixture of several materials on ground. Spectral unmixing algorithms try to quantify the fractions of pure spectra (or endmembers) within a mixed pixel. Nevertheless, such algorithms rely on the assumption that it is possible to identify a pure pixel for all the classes of interest within the image, which is not always the case.

This paper introduces two novel techniques in hyperspectral image processing which try to overcome the described problems by means that could be thought as opposite to each other. On the one hand, a semi-automated process using sparse approximation and derivative features is presented, which identifies the classes of interest within an image by using an external overcomplete spectral library. On the other hand, a classification approach for hyperspectral data based on synergetics theory analyzes the image within a user-defined subspace, which helps at focusing on the relevant information for a given classification task. The output of the first algorithm can be used to build a subspace for the second, thus achieving a semi-automatic classification of the data, carried out in a "semantic" subspace, i.e. a subspace in which each dimension is related to a class of interest.

\section{ENDMEMBER DETECTION USING SPARSE APPROXIMATION AND DERIVATIVE METHODS}

The identification of classes of interest in a hyperspectral image is a non-trivial issue affecting most hyperspectral analysis algorithms, including the synergetics-based classification described in section 3. With the aim of providing an accurate classification map with ideally no supervision, we introduce a semi-automated selection of the materials present within an image based on sparse approximation and derivative spectroscopy.

We consider to have available a spectral library containing in situ measured spectra of targets. As the number of entries in the spectral dictionary is higher than the dimensionality of the image, the spectral library is overcomplete and the estimated coefficient vector for each image element is highly sparse. Under this condition, we 
determine the best combination of endmembers using a sparse approximation method [3], drastically reducing the dimensionality of the spectral library by considering only relevant entries. As the algorithm works with external spectral libraries, no expensive learning or endmember selection steps are needed. Being that the library elements are highly correlated, it is difficult to correctly detect them in an image. Such coherence, as well as the dependence of the scene from the illumination conditions, is decreased by analyzing the spectra derivatives, both in the spectral library and in the image. The use of derivative spectroscopy for resolving the fine structures of overlapping spectral features is well known, as absorption features are sharpened while broad features are suppressed in the process [4]. Assuming that any mixed pixel consists of linearly mixed materials, the absorption features exhibited by the pure spectra will be discernible in the mixed spectra.

The proposed algorithm was tested on a 500 x 500 urban scene acquired over the city of Munich, Germany by the hyperspectral HyMAP sensor, with 125 bands and a spatial resolution of $4 \mathrm{~m}$. The spectral library employed contains 160 spectra identifying the same number of materials [5]. An abundance map for red bricks produced with the proposed method and a comparison with the result obtained using the original spectra are reported in Fig. 1.

\section{HYPERSPECTRAL IMAGE CLASSIFICATION BASED ON SYNERGETICS}

This section introduces a classification methodology for hyperspectral data based on synergetics theory, in which the subspace on which the data are projected is defined by the user. Synergetics is a two decade old theory describing the spontaneous formation of patterns and structures in a system through self-organization [6]. Applications based on synergetics have been derived in the pattern matching and image classification domains $[7,8]$, but they have often been limited by the dependency of such systems on scaling, rotation and shifting of the images [6]. These drawbacks can be discarded in applications to hyperspectral data performed in the spectral domain, as each image element is analyzed separately, and is represented as a data point projected in a subspace composed by a set of user-defined prototype vectors, belonging to some classes of interest. The pixel may then be represented as a particle on a potential surface, built as a manifold in this subspace. After resolving the dynamics of its movement on the surface, the pixel is attracted by one of several possible final states, with each one being associated with a user-defined class, and hence classified (Fig. 2). Such approach could be considered similar to Orthogonal Subspace Projection [9], but an important difference is that synergetics theory ensures that a solution always exists for each image element and that it is unique.

As typical synergetics-based systems have the drawback of a rigid training step, we modify it to allow the selection of user-defined training areas, used to weight the prototype vectors through attention parameters: if $n$ samples are available for any given class, $n$ independent classifications are obtained on the basis of a single training sample per class, with the rest of the training set employed to shape the potential surface of Fig. 2 in order to minimize false alarms for each single classification. A final classification map is produced through majority vote of the independent classifiers. As the majority vote improves final results only if the single classifiers are accurate enough, the proposed technique yields better results when employed on natural scenes (e.g. vegetation or mineralogy applications), as it is not able to capture relevant intra-class variations.

Fig. 3 shows a sample classification based on synergetics, performed on the AVIRIS Salinas dataset, composed of 512 x 217 samples with 192 spectral bands, with the water absorption bands removed as in [10]. The overall classification accuracy (OA), obtained on the basis of only 20 training samples per class, is $\mathrm{OA}=85.41 \%$, while the average accuracy per class (AA) is $\mathrm{AA}=89.81 \%$. These results are comparable to the ones obtained through Neural Networks with a larger training set in [10] $(\mathrm{OA}=87.55 \%$, $\mathrm{AA}=88.04 \%)$.

\section{COMBINING BOTH METHODS: EXPERIMENTAL RESULTS}

The methods presented in Sections 2 and 3 can be effectively combined to derive a semi-automated endmember detection and classification procedure. We analyze a subset of the AVIRIS Cuprite dataset, composed of 200 x 300 samples with 184 spectral bands (water absorption bands removed). The data are represented as reflectance values, in order to allow a direct comparison with the Aster spectral library 2.0 [5]. The library, containing 813 spectra belonging to natural and man-made materials, has been used as input to estimate the content of the scene. All spectra in the library and all image elements are first normalized, so that their Euclidean norm is equal to 1. Derivatives features are then extracted from both the laboratory spectra and from the image, and used as input for the endmember detection algorithm. The method found 23 materials in the scene, most of which belonging to minerals widely acknowledged to be present in the area. In a second step, the 10 purest pixels for each class have been selected and fed to a synergetics-based classifier, as briefly described in previous section, resulting in 10 independent classifications, which have been then combined in a single one on the basis of a simple majority vote.

Results in Fig. 5 show the detected areas for some materials: the map agrees with the general interpretation of the area, as far as the location of alunite and kaolinite sites is concerned [11]. The site is also known to contain jarosite and muscovite: the latter justifies the presence of aluminium, as it is mostly composed of this metal. 

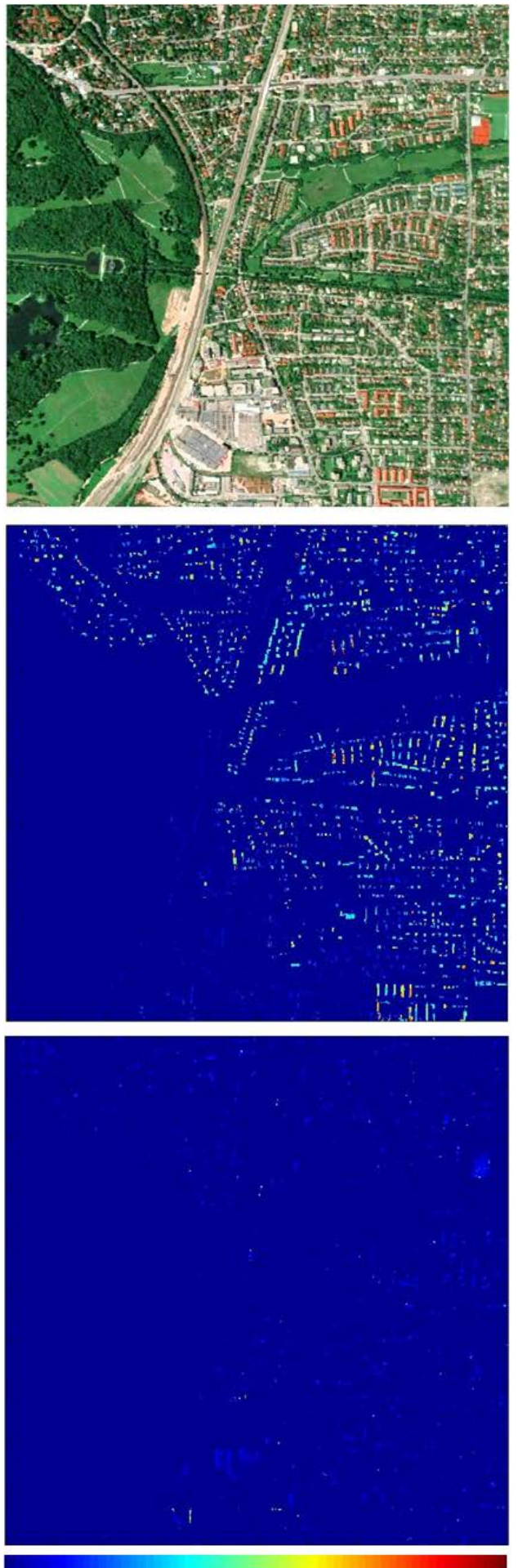

Low

High

Figure 1. Red brick abundance estimation. From top down: HyMAP test image (RGB composition), estimation using derivative spectra, and estimation using standard spectra.

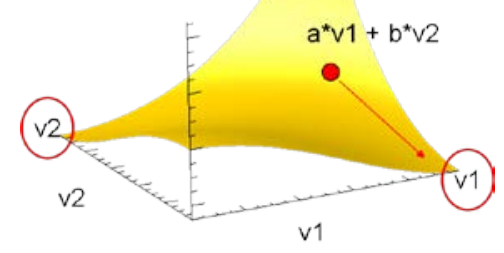

Figure 2. Principle of synergetics-based classification. An object is represented as a linear combination of two test vectors. It is then projected onto a potential surface, and classified as it is attracted by one of the possible final states, which are associated to classes of interest.
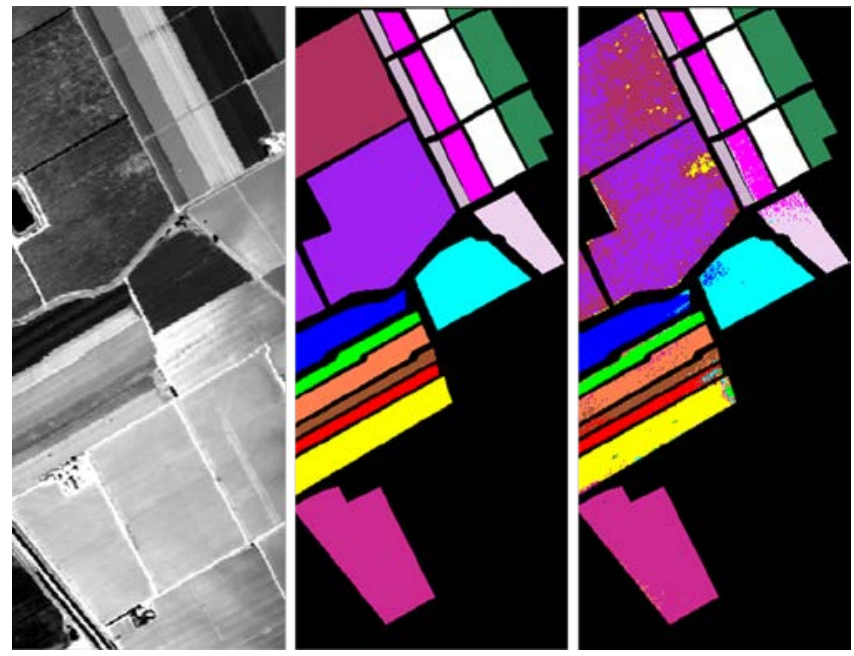

Figure 3. From left to right: sample band from the Salinas dataset, available ground truth, and classification results based on synergetics.

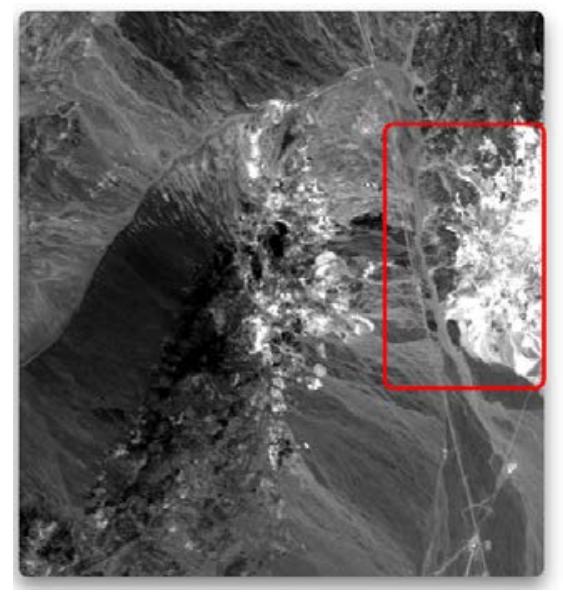

Figure 4. Sample band from a subset of the AVIRIS Cupirite dataset, with the area analyzed in Fig. 5 marked in red. 


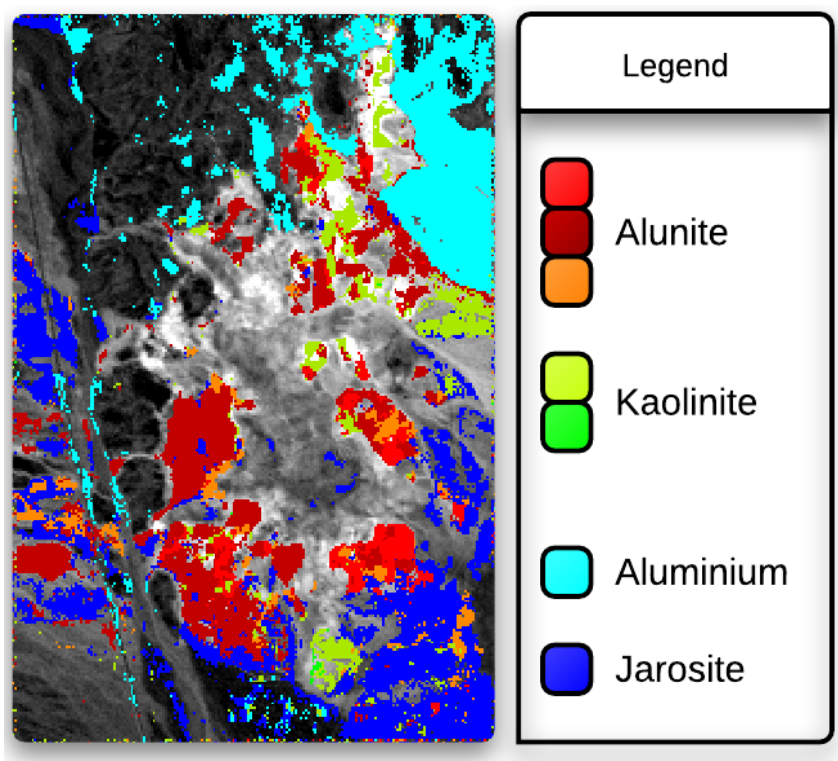

Figure 5. Classification based on synergetics for the area in Fig. 4 for some endmembers, automatically selected from the spectral library [5] by unmixing based on sparse approximation without any prior knowledge as input.

\section{CONCLUSIONS}

In this paper we presented two analysis algorithms for hyperspectral data, which could be employed together in an effective and quasi-unsupervised workflow for classification purposes, also at sub-pixel level.

An automatic endmember detection algorithm is presented to identify the materials within an image. Derivative spectroscopy is used to highlight absorption features in the image, and to achieve illumination independence to some degree. Subsequently sparse approximation is chosen as a robust estimation method, if overcomplete dictionaries are employed. This approach can be used to find the relevant classes to be used by the synergetics-based classifier, and can represent a good alternative for detailed studies on material detection, as well as for creating mixed pixel maps, whenever a high number of materials is involved.

A focused dimensionality reduction is then achieved through the introduced classification methodology based on synergetics theory, which represents the data in a "semantic" vectorial space. Such user-defined subspace uses a basis derived from the classes of interest chosen by the previous algorithm, which are selected as prototype vectors. The results obtained are comparable to state of the art classification methodologies, and could be easily improved by taking into account the spatial distribution of the data, through the application of morphological filtering or segmentation. The high degree of automatism of the proposed technique justifies its practical use in applications to natural scenes, for which the homogeneity of the materials causes both the endmember extraction algorithm and the classifier to produce more accurate results.

\section{REFERENCES}

[1] J. Bioucas-Dias and J. Nascimento, "Hyperspectral subspace identification”, IEEE Tr. Geosci. Rem. Sensing, vol. 46, no. 8, pp. $2435-2445,2008$.

[2] S. Kaewpijit, J. Le Moigne, and T. El-Ghazawi, "Automatic reduction of hyperspectral imagery using wavelet spectral analysis”, Tr. Geosci. Rem. Sensing, vol. 41, no. 4, pp. 863-871, 2003.

[3] B. Efron, T. Hastie, I. Johnstone, and R. Tibshirani, "Least Angle Regression”, Annals of Statistics, vol. 32, no. 2, pp. 407499, 2004.

[4] G. Talsky, "Derivative Spectrophotometry of First and Higher Orders”, VCH Verlagsgesellschaft mbH, 1994.

[5] A. M. Baldridge, S. J. Hook, C. I. Grove, and G. Rivera, "The aster spectral library version 2.0,” Remote Sensing of Environment, vol. 113, no. 4, pp. 711-715, Apr. 2009.

[6] H. Haken, H., "Synergetics Computers and Cognition", Springer Series Synergetics, vol. 50, 1991.

[7] T. Hogg, T. Talhami, and D. Rees, "An improved synergetic algorithm for image classification”, Pattern Recognition, vol. 31, no. 12 , pp. 1893 - 1903, 1998.

[8] K. Crounse and L. Chua, "A synergetics approach to image processing in cellular neural networks", IEEE International Symposium on Circuits and Systems ISCAS '96, vol. 3, pp. 134 137, 1996.

[9] C. Chang, "Orthogonal subspace projection (OSP) revisited: a comprehensive study and analysis," IEEE Tr. Geosci. Rem. Sensing, vol.43, no.3, pp. 502- 518, 2005.

[10] A. Plaza, P. Martinez, J. Plaza, and R. Perez, “Dimensionality reduction and classification of hyperspectral image data using sequences of extended morphological transformations”, IEEE Tr. Geosci. Rem. Sensing, vol. 43, no. 3, pp. 466-479, 2005.

[11] J. P. Hoffbeck and D. A. Landgrebe, "Classification of High Dimensional Multispectral Image Data”, $4^{\text {th }}$ JPL Airborne Geoscience Workshop, 1993. 